\title{
Fabrication of 3D cell-laden hydrogel microstructures through photo-mold patterning
}

\author{
P Occhetta ${ }^{1}$, N Sadr ${ }^{2}$, F Piraino ${ }^{1}$, A Redaelli ${ }^{1}$, M Moretti ${ }^{2,3}$ and M Rasponi ${ }^{1,3,4}$ \\ ${ }^{1}$ Department of Bioengineering, Politecnico di Milano, Milano, Italy \\ ${ }^{2}$ Cell and Tissue Engineering Lab, IRCCS Istituto Ortopedico Galeazzi, Milano, Italy \\ E-mail: marco.rasponi@polimi.it
}

\begin{abstract}
Native tissues are characterized by spatially organized three dimensional (3D) microscaled units which functionally define cells-cells and cells-extracellular matrix interactions. The ability to engineer biomimetic constructs mimicking these 3D microarchitectures is subject to the control over cell distribution and organization. In the present study we introduce a novel protocol to generate 3D cell laden hydrogel micropatterns with defined size and shape. The method, named Photo-Mold Patterning (PMP), combines hydrogel micromolding within polydimethylsiloxane (PDMS) stamps and photopolymerization through a recently introduced biocompatible ultraviolet (UVA) activated photoinitiator (VA-086). Exploiting PDMS micromolds as geometrical constraints for two methacrylated prepolymers (Polyethylen Glycol Diacrylate and Gelatin Methacrylate), micrometrically resolved structures were obtained within a 3 minutes exposure to a low cost and commercially available UVA LED. The PMP was validated both on a continuous cell line (Human Umbelical Vein Endhotelial Cells expressing Green Fluorescent Protein, HUVEC GFP) and on primary human Bone Marrow Stromal Cells (BMSCs). HUVEC GFP and BMSCs were exposed to 1.5\% w/v VA-086 and UVA light $(1 \mathrm{~W}, 385 \mathrm{~nm}$, distance from sample $=5 \mathrm{~cm})$. Photocrosslinking conditions applied during the PMP did not affect negatively cells viability or specific metabolic activity. Quantitative analyses demonstrated the potentiality of PMP to uniformly embed viable cells within 3D microgels, creating biocompatible and favorable environments for cell proliferation and spreading during a 7 days culture. PMP can thus be considered as a promising and cost effective tool for designing spatially accurate in vitro models and, in perspective, functional constructs.
\end{abstract}

This is the peer reviewed version of the following article: P. Occhetta, N. Sadr, F. Piraino, A. Redaelli, M. Moretti and M. Rasponi, Biofabrication, 2013, 5, 035002, which has been published in final form at [http://iopscience.iop.org/17585090/5/3/035002]. IOP Publishing open access policy.

\footnotetext{
${ }^{3}$ Authors contributed equally.

${ }^{4}$ Author to whom any correspondence should be addressed.
} 


\section{Introduction}

Tissues in the body are composed of functional three-dimensional (3D) units, characterized by a spatially organized microarchitecture comprised of cells and extracellular matrix (ECM) [1]. Each cell continuously interacts with its surrounding 3D microenvironment through biochemical, biomechanical and bioelectrical signals, which vary dynamically in both time and space and contribute in the regulation of cellular behavior and fate processes [2]. When developing new in vitro models or functional bio-constructs, the ability of spatially and functionally replicating the native tissue microarchitecture is a crucial step to obtain reliable and physiologically consistent cell responses [3]. Indeed, while traditional 2D in vitro culture techniques lack in reproducing the complexity found in vivo, 3D models have been demonstrated to recapitulate unprecedented cues from the native environment [4]. Nevertheless, only few recent technological approaches succeeded in tailoring the microscale 3D geometrical features of cell microenvironment [5]. Among other methods, the combination of novel biomaterials and microfabrication technologies has been rapidly generating perspectives for addressing this challenge [6-8].

The choice of appropriate biomaterials becomes crucial in order to mimic native tissues with respect to both biological integration and geometrical replication; these requirements can be satisfied by using polymeric hydrogels[9]. Indeed, hydrogels exhibit high similarities to the natural ECM [10] thanks to some unique properties, such as highly swollen network structures, which maximize nutrients transfers, the presence of functional groups, which can be chemically modified to add specific functions, and mechanical properties easily tunable within a wide range [11]. Moreover, they have been demonstrated to be highly affine with innovative microtechnologies such as photo and soft lithographic approaches [12], making them suitable to obtain 3D biomimetic replicates with a highly controlled microtopography. Hydrogel microstructures have been widely used either as substrates for cellular seeding [13] or matrices for direct cell encapsulation [14, 15]. The possibility to obtain cell-laden hydrogel derives in particular from the biocompatibility of many crosslinking approaches. For example, thermal hydrogel reticulation has been investigated in combination with inexpensive soft lithography techniques, resulting in micropatterned 3D structures with dimension ranging between $1000 \mu \mathrm{m}$ [16] and $50 \mu \mathrm{m}$ [17]. However, the presence of cells within the biomaterial de facto limits the applicable temperature range [18], which can result in relatively long crosslinking reaction times. Fast crosslinking reaction corresponds to rapid gel viscosity increase, a characteristic that compensates cells tendency to settle down and favors a uniform 3D cell distribution within the microstructures. Thus, the temporal control over reaction represents a critical parameter to generate uniformly populated 3D constructs. For this purpose, additional rotating systems have been used to thermally crosslink cell-laden hydrogels [19]. As an alternative, light responsive hydrogels (or photohydrogels) have been successfully used to obtain 3D patterns within tens of seconds [20]. Photopatterning techniques are generally based on the presence of a photomask between the light source and the pre-polymer solution, the latter including light-specific photoinitiator (PI) molecules [21]. Based on this approach, the fabrication of free standing microgels [22] as well as micropatterns [23] has been demonstrated. In addition, photopatterning was iterated for the construction of multicellular patterns [24] as well as the free standing microgels were assembled based on different 
interaction forces (interface based, magnetic, acoustic) to form complex macrostructures [25]. Similarly to photolithography, the achievable resolution of microgels obtained with photopatterning approaches is strongly affected by light source collimation [26]. At the same time, light source emission spectrum together with PI chemistry can severely affect the biocompatibility of the method. Irgacure 2959 is currently the most commonly used PI molecule for the generation of viable cell-laden constructs, due to its low level of cytotoxicity [27-29]. However, Irgacure 2959 presents an absorption peak in a wavelength range between 250 and $300 \mathrm{~nm}$ [30], which is known to induce significant cell mortality even at relatively low level of absorbed energy [31]. The search for photoinitiators minimizing ultraviolet (UV) light exposure as well as molecule cytotoxicity led to investigate alternative solutions [32]. Among others, VA-086, a water soluble azo initiator molecule, recently proved to yield low cytotoxic effects in both precursor and radical forms [33], together with an absorption peak, corresponding to a range of wavelengths between 365 and 385nm, more conservative in terms of cell viability.

With the aim of obtaining 3D uniformly dispersed cell-laden micropatterns replicating user defined geometrical features we present a simple microscaled Photo-Mold Patterning (PMP) protocol. This protocol combines the advantages of thermal mold patterning approaches (high resolution without the need of expensive collimated light sources) [16] with the benefits of using photopolymerizable hydrogels (reduced crosslinking time) [14]. In addition, thanks to the use of a recently introduced biocompatible PI molecule (VA-086), we proved for the first time the possibility to generate highly viable cell-laden micropatterns through a low-cost and versatile UVA LED light source. This method is thus accessible to a wide range of laboratories and budgets.

\section{Materials and methods}

A novel Photo-Mold Patterning (PMP) protocol for hydrogel microstructuring was developed in this study. Briefly, a polydimethylsiloxane (PDMS) microfluidic chip with geometrically defined micropatterns was placed against a glass substrate and used as mold, hosting cell suspensions of pre-polymer light-sensitive solution. UV light was provided through the PDMS layer until complete crosslinking. Once the stamp was removed, cell-laden microstructures were incubated with culture medium and cultured for 7 days.

\subsection{Photo-Mold Patterning (PMP) protocol}

The layouts of the desired hydrogel micropatterns were designed through a CAD software (AutoCAD, Autodesk Inc.) and the corresponding master molds were realized through standard photolithography techniques [34]. Stamps were produced by replica molding on the master molds by casting PDMS in a ratio 10:1 w/w (pre-polymer to curing agent), degassing and curing at $80^{\circ} \mathrm{C}$ for 3 hours. The PDMS stamps were trimmed so to show microchannel openings on their lateral sides and subsequently air-plasma treated to induce surface hydrophilicity. The PMP devices were finally assembled by placing the stamps against histology glass slides, which were previously treated with 3-(trimethoxysilyl) propyl methacrylate (TMSPMA) (SigmaAldrich $^{\circledR}$ ) to enhance hydrogel adhesion [35]. 
The photoinitiator molecule 2,2'-Azobis[2-methyl-N-(2-hydroxyethyl)propionamide] (VA-086, Wako Chemicals $\mathrm{GmbH}$ ) was added at $1.5 \%$ w/v to a phosphate buffer saline (PBS) solution. Subsequently, $10 \%$ w/v methacrylated polymer precursor was included and mixed at room temperature until complete dissolution. The solution was used to suspend cells at the desired final concentration. In this study, two polymer precursors were considered: a commercially available polyethylen glycol diacrylate (PEGDA, Sigma- Aldrich ${ }^{\circledR}$ ) and gelatin methacrylate (GelMA) synthesized following a previously reported method [36, 37]. Through a standard pipettor, $2.5 \mu \mathrm{l}$ of the cell-laden pre-polymer solution were placed in contact with a PMP device opening and allowed to fill the corresponding microchannel driven by capillary forces. Once completely filled, the sample was irradiated ( 3 minutes) using a $1 \mathrm{~W}$ LED (385nm; LZ4-00UA00, LED Engin, Inc.) placed at 5 $\mathrm{cm}$ from samples. Subsequently the PDMS mold was removed leaving the hydrogel micropattern adherent on the glass slide. The sequence of operations for the PMP protocol is depicted in figure 1 .

\section{Chip assembly}

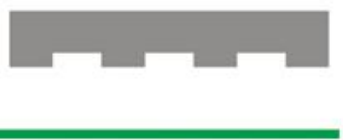

(a)
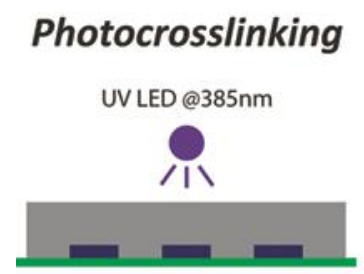

(c)

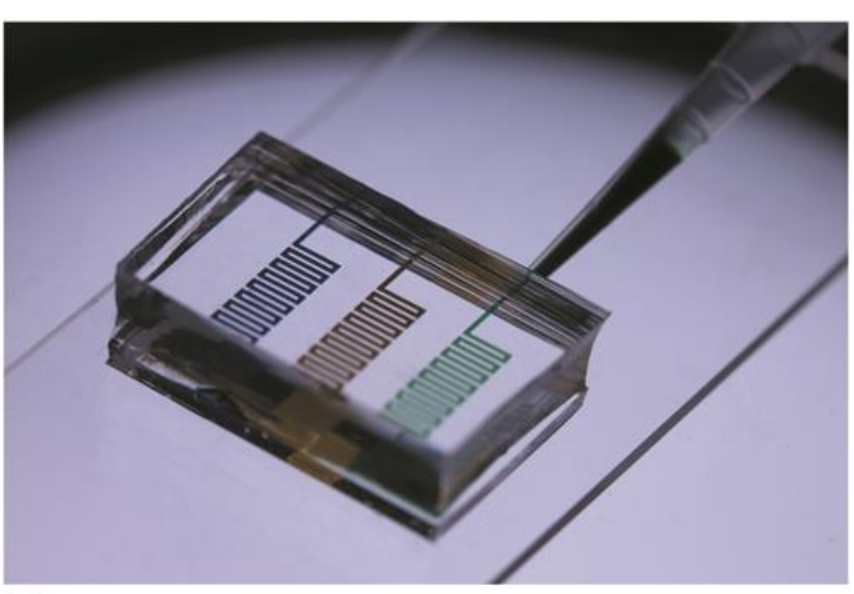

(e)

Figure 1. Photo-Mold Patterning (PMP) protocol. (a) The PDMS microfluidic mold is placed against a 3(trimethoxysilyl) propyl methacrylate (TMSPMA) treated slide. (b) Hydrogel prepolymer is injected into the channels, and (c) the photopolymerization is achieved using a commercial LED emitting at $385 \mathrm{~nm}$. (d) Upon PDMS removal, the 3D patterns are revealed on the glass substrate. (e) An example of PMP is reported, where three mold channels were independently filled with different colors. 
To evaluate the PMP ability to reliably achieve micropatterns, two chip layouts were considered for both PEGDA and GelMA hydrogels. In particular, a straight channel and a serpentine configuration were conceived, both having channel width of $250 \mu \mathrm{m}$ and height of $80 \mu \mathrm{m}$.

\subsection{Cell Sorts and Expansion}

Immortalized human umbilical vein endothelial cells constitutively expressing green fluorescent protein (HUVEC GFP; a generous gift from Dr. J. Folkman, Children's Hospital, Boston), were maintained in Endothelial Cells Growth Medium MV2 (Promocell GmbH) enriched with SupplementMix (Promocell $\mathrm{GmbH}$ ) containing $1 \%$ antibiotic (penicillin/streptomycin, Gibco ${ }^{\circledR}$ ) and were passaged when $70-80 \%$ confluence was reached while medium was changed every 4 days.

Bone marrow aspirates were obtained from donors during routine orthopedic surgical procedures. Bone Marrow Stromal Cells (BMSC) were isolated by adherence. Freshly isolated cells were plated at a density of $1 \times 10^{5}$ nucleated cells $\mathrm{cm}^{-2}$ and cultured overnight to allow cell adhesion. Non adherent cells, including those of the hematopoietic lineage, were then removed. Cell expansion was carried out in complete medium which consisted of $\alpha$-Modified Eagle's Medium, 10\% fetal bovine serum, 10mM HEPES, $1 \mathrm{mM}$ sodium pyruvate, $100 \mathrm{U} \mathrm{ml}^{-1}$ penicillin, $100 \mu \mathrm{g} \mathrm{ml}^{-1}$ streptomycin and $292 \mu \mathrm{g} \mathrm{ml}^{-1} \mathrm{~L}$-glutamine (all GIBCOß) supplemented with $5 \mathrm{ng} \mathrm{ml}^{-1}$ fibroblast growth factor-2 (Peprotech). Medium was changed every 4 days. The cells were harvested after 7-10 days and seeded at lower density $\left(3 \times 10^{3}\right.$ cells $\left.\mathrm{cm}^{-2}\right)$, and passaged when 70-80\% confluence was reached. For the experiments described in this study BMSCs were collected at the third passage.

Both cell types were cultured in a standard cell culture incubator in a $5 \% \mathrm{CO}_{2}$ atmosphere at $37^{\circ} \mathrm{C}$.

\subsection{PMP Cytotoxicity Analysis}

To assess possible cytotoxic effects of factors used during the PMP protocol, experiments were carried out on 2D cultures. For this purpose, cells were seeded at a density of $10 \times 10^{3}$ and $3 \times 10^{3}$ cells cm $\mathrm{cm}^{-2}$ for HUVEC GFP and BMSCs, respectively. Samples with identical cell numbers were frozen in order to be used as DNA reference. Right after seeding, cells still in suspension were subjected to different photocrosslinking conditions (as described in Table 1). No stimuli were applied in the condition used as control. Independently from the duration of the treatment, cells were allowed to adhere for a period of 4 hours before medium change. After 24 hours under standard culture conditions, each sample was analyzed in terms of metabolic activity: cells were incubated for 4 hours in AlamarBlue 10\% v/v solution (Invitrogen) and the absorbance was detected using a spectrophotometer at $570 \mathrm{~nm}$. The number of cells contained in each well was evaluated. Samples were frozen at $-80^{\circ} \mathrm{C}$ overnight to promote cell lysis and therefore DNA extraction. DNA quantification was performed by means of a commercially available fluorescence based kit, namely CyQUANT ${ }^{\circledR}$ Cell Proliferation Assay (Invitrogen). Working solutions were prepared according to the manufacturer's protocols. Sample fluorescence intensity, detected with a spectrophotometric approach $(485 / 538 \mathrm{~nm})$, was associated to 
cell DNA content through a calibration curve. The reference samples, containing the number of cells seeded at the initial time point were used to normalize DNA content in each sample and calculate cell number per sample. Finally, for each sample the absorbance value relative to the metabolic activity was normalized to the cell number and used as indicator for the specific cellular metabolic activity.

Table 1 summarizes the conditions tested, eliciting from combinations of: PI type, PI exposure time and light source exposure time. In particular, the cytotoxicity of the VA-086 was compared to that induced by the more commonly used Irgacure 2959 [38]. Two solutions were prepared by dissolving VA-086 (Wako Chemicals $\mathrm{GmbH}$ ) and Irgacure 2959 (Ciba Chemicals) in culture medium at 1.5\% w/v and 0.05\% w/v, respectively (due to different photoinitiator activities) [33]. The solutions were then used to suspend cells at the target concentrations. PI molecule were either removed after 4 hours (short exposure, SE conditions) or left in solution until the end of the experiment (long exposure, LE conditions). The effect of UV light was assessed both individually and in combination with PI molecule: three minutes exposure to $1 \mathrm{~W} 385 \mathrm{~nm}$ LED was applied to cells in standard culture medium (UV condition) and cells in 1.5\% w/v VA-086 culture medium (UV + VA-086 condition), respectively. The exposure time of 3 minutes was chosen as the photopolymerization time required in the PMP to obtain the 3D micropatterns. For each cell type, five replicates were evaluated for each condition tested $(n=5)$.

Table 1. Scheme of the conditions tested on HUVEC GFP and BMSC to assess UV light and PI effect on cell viability.

\begin{tabular}{cccc}
\hline $\begin{array}{c}\text { Condition } \\
\text { name }\end{array}$ & $\begin{array}{c}\text { PI } \\
\text { (exposure time) }\end{array}$ & $\begin{array}{c}\text { Light Source } \\
\text { (exposure time) }\end{array}$ & $\begin{array}{c}\text { Photocrosslinking condition } \\
\text { tested }\end{array}$ \\
\hline CTRL & - & - & No stimuli \\
SE VA-086 & VA-086 $(4 \mathrm{hr})$ & - & PI (short exposure) \\
LE VA-086 & VA-086 $(24 \mathrm{hr})$ & - & PI (long exposure) \\
SE Irg-2959 & Irgacure $2959(4 \mathrm{hr})$ & - & PI (short exposure) \\
LE Irg-2959 & Irgacure 2959 $(24 \mathrm{hr})$ & - & PI (long exposure) \\
UV & - & $\lambda=385 \mathrm{~nm}(3 \mathrm{mins})$ & UV light \\
UV + VA-086 & VA-086 $(4 \mathrm{hr})$ & $\lambda=385 \mathrm{~nm}(3 \mathrm{mins})$ & Combined UV light and PI \\
\hline
\end{tabular}

\subsection{Cell encapsulation and distribution analysis}

HUVEC GFP were encapsulated within GelMA through the PMP protocol, and the resulting micropatterns were analyzed in terms of 3D cell distribution. Cells were trypsinized, counted and resuspended at different initial concentrations $\left(1,5\right.$ and $\left.10 \times 10^{6} \mathrm{cells} \mathrm{m}^{-1}\right)$ into $10 \% \mathrm{w} / \mathrm{v}$ GelMA containing $1.5 \% \mathrm{w} / \mathrm{v}$ VA-086 prepolymer solution and the PMP protocol was performed as previously described leading to cell-laden micropatterns formation. Specifically, the range of initial cell densities were considered in the perspective to obtain constructs targeting tissues with different cellularity. Immediately after the photopolymerization, micropatterns were treated sequentially with $4 \%$ paraformaldehyde and $0.1 \%$ Triton-X to fix and permeabilize cells, followed by DAPI (Invitrogen) to fluorescently stain cell nuclei. Samples were thus mounted on slides 
and observed by fluorescent confocal microscopy (Leica TCS SP8). The 3D position of cell nuclei was analyzed with a semi-automated object 3D counter plugin of the NIH ImageJ software, using an intensitybased algorithm and verified in $10 \%$ microscope fields by manual counts. To assess the cell distribution along the channel length, three equidistant channel areas $(l=1 \mathrm{~mm})$ were chosen in correspondence to the inlet, center and outlet regions. Each area was then divided along its width and height in ten $(\mathrm{w}=25 \mu \mathrm{m})$ and four $(\mathrm{h}=20 \mu \mathrm{m})$ bands, respectively. The number of cells present in each region of interest was evaluated to quantify the cell distribution in both lateral and vertical channel dimensions. For each cell concentration tested, three samples of the straight channel configuration were considered $(n=3)$.

\subsection{Cell culture within hydrogel micropatterns}

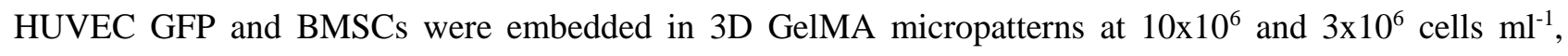
respectively, and cultured up to 7 days $[16,23]$. This biological validation was only carried on GelMA, being PEGDA not suitable for cell adhesion in its native form [39]. For this purpose, cells were trypsinized, counted and resuspended into $10 \% \mathrm{w} / \mathrm{v}$ GelMA containing $1.5 \% \mathrm{w} / \mathrm{v}$ VA-086 prepolymer solution and the PMP protocol was performed as previously described. The glass slides containing cell-laden microgels were gently washed with PBS to remove possible unreacted PI molecules, incubated under standard culture conditions and cell behavior was evaluated at days 1,3 and 7. For each time point, the micropatterns were treated sequentially with $4 \%$ paraformaldehyde and $0.1 \%$ Triton-X to fix and permeabilize cells, followed by phalloidin (BODIPY ${ }^{\circledR} 558 / 568$ phalloidin, Invitrogen) and DAPI (Invitrogen) to fluorescently stain respectively filamentous F-actin and cell nuclei. Samples were thus mounted on slides and observed by fluorescent confocal microscopy (Leica TCS SP8).

\subsection{Statistical Analyses}

Data are expressed as mean \pm standard deviation and statistical analyses (one-way ANOVA) were performed using GraphPad Prism v5.00 (GraphPad Software, San Diego, CA).

\section{Results}

PDMS soft lithography and a UV activated photoinitiator (VA-086) were combined to draw a novel Photomold Patterning (PMP) protocol, obtaining 3D hydrogel micropatterns upon UVA light exposure.

To assess the versatility of the PMP approach in terms of geometry and materials, two geometrical configurations were reproduced and two different polymer precursors were used, a synthetic and a naturally derived one. An exposure time of 3 minutes to UVA LED, combined with a $1.5 \% \mathrm{w} / \mathrm{v}$ PI concentration allowed to achieve highly defined micropatterns, narrowly replicating $250 \mu \mathrm{m}$ width and $80 \mu \mathrm{m}$ height PDMS mold features for both the configurations (figure 2). Moreover, the PMP protocol was demonstrated to be applicable to both the materials tested, leading to comparable results in terms of 3D geometry replication. As shown in figure 2(e) and (f) even using a non-collimated light source, the cross sections obtained from both PEGDA and 
GelMA resulted squared, replicating the PDMS channels layout without any magnification of patterns width [26].

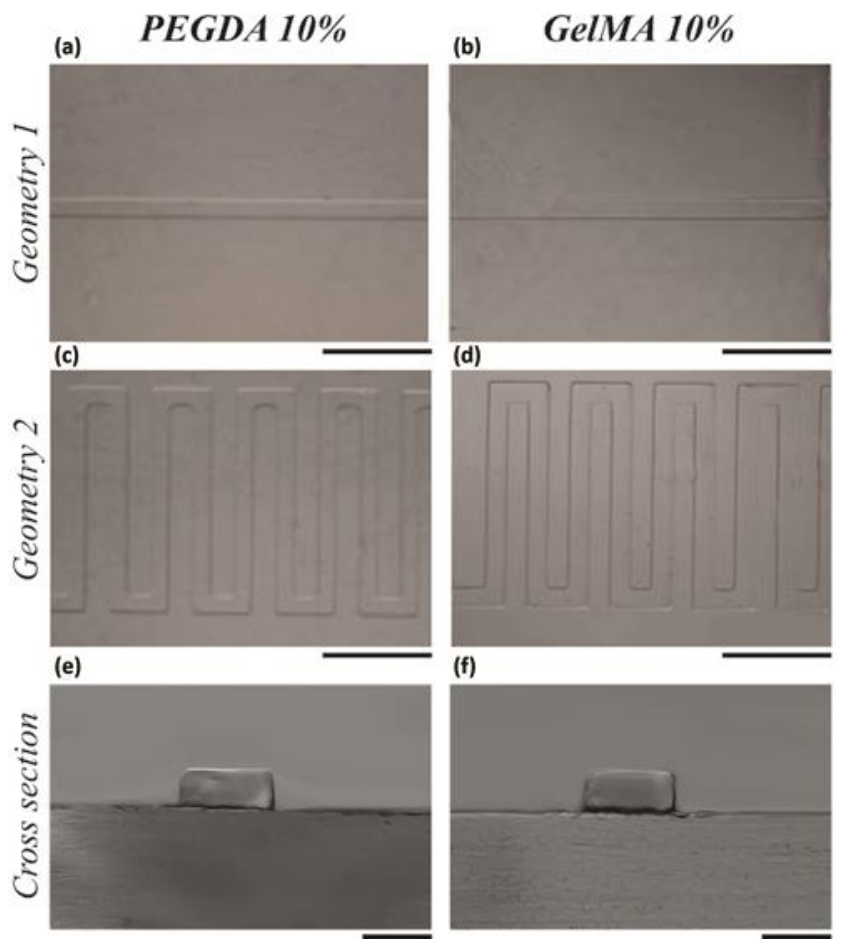

Figure 2. 3D replication of different geometries through PMP. Phase images of hydrogels obtained from $10 \% \mathrm{w} / \mathrm{v}$ PEGDA ((a), (c) and (e)) and 10\% w/v GelMA ((b), (d) and (f)) polymer precursors upon a 3 minutes UVA exposure in the presence of $1.5 \%$ w/v VA-086. (a)-(d) PMP allowed to achieve micrometrically defined patterns, narrowly replicating $250 \mu \mathrm{m}$ width and $80 \mu \mathrm{m}$ height mold features for both the configurations and the materials tested. (Scale bars: $1000 \mu \mathrm{m}$ ). (e)-(f) The hydrogel cross sections obtained from both PEGDA and GelMA resulted squared, exactly reproducing the PDMS channel lateral layouts. (Scale bars: $200 \mu \mathrm{m}$ ).

\subsection{Photocrosslinking cytotoxicity analysis}

To assess the cytocompatibility of the Photo-mold Patterning (PMP) protocol, the behavior of HUVEC GFP and BMSCs was tested in response to different photocrosslinking conditions. The results shown in figure 3 are referred to a 24 hours culture period, chosen to reasonably neglect the cell proliferation as cause of variations in cell number or specific metabolic activity.

The effect of long and short exposure to photoinitiator inactive molecules was first analyzed, by comparing the PI used in the presented protocol (VA-086) with the widely used Irgacure-2959 [27]. Comparable results in terms of cell viability were obtained with both the investigated PIs as detailed in figure 3(a) and (b). VA-086 in a 30-fold higher mass concentration than Irgacure-2959 (1.5\% w/v compared to 0.05\% w/v) didn't significantly affect cell viability and specific metabolic activity compared to control conditions after a 4 hours exposure time. A long exposure to VA-086 caused a decrease in HUVEC GFP number, though maintaining a high specific metabolic activity comparable both to control and LE to Irgacure conditions. This can however be considered an extreme case, since for the presented PMP protocol the cell exposure to VA086 is limited to the photopolymerization time ( 3 minutes).

Being VA-086 molecules activated at a wavelength range of $365-385 \mathrm{~nm}$, the cytocompatibility of a 385nm LED light source was also assessed (UV condition). The sole UV irradiation did not cause any 
significant decrease on cells viability nor metabolic activity after a $3 \mathrm{~min}$ exposure time compared to the conditions in absence of irradiation (figure 3(a), (b), (d) and (e)). Finally, the combination of UV and VA-086 was considered to replicate the stimuli applied during the PMP protocol. Again, treatment with UV and VA$086(3 \mathrm{~min} ; 1.5 \% \mathrm{w} / \mathrm{v})$ did not show any statistically significant reductions on cell number and metabolic activity compared to the control conditions (figure 3(c) and (f)) and was, therefore, used for subsequent experiments.

\section{Cell number quantification}
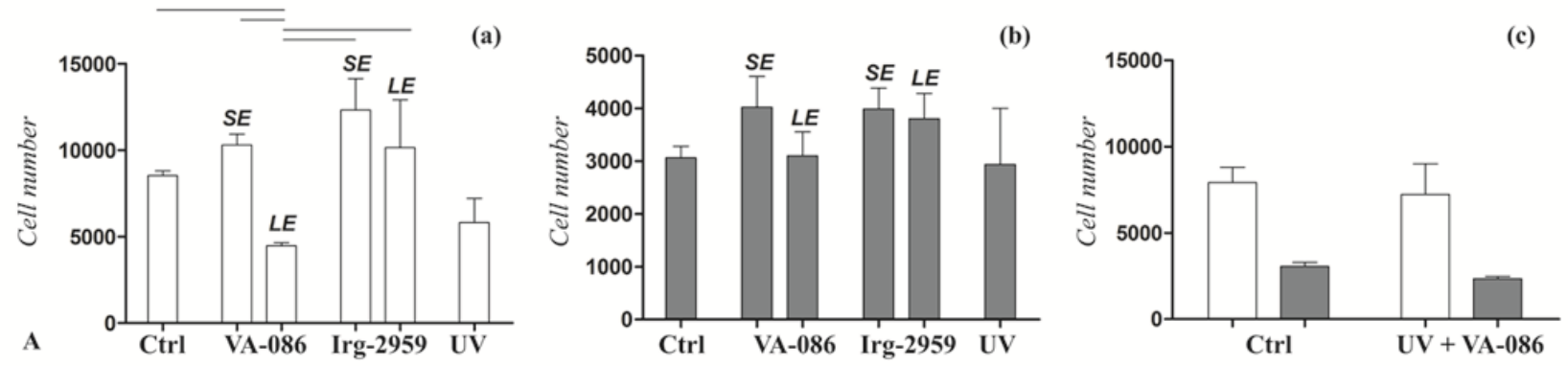

\section{Specific Metabolic Activity}
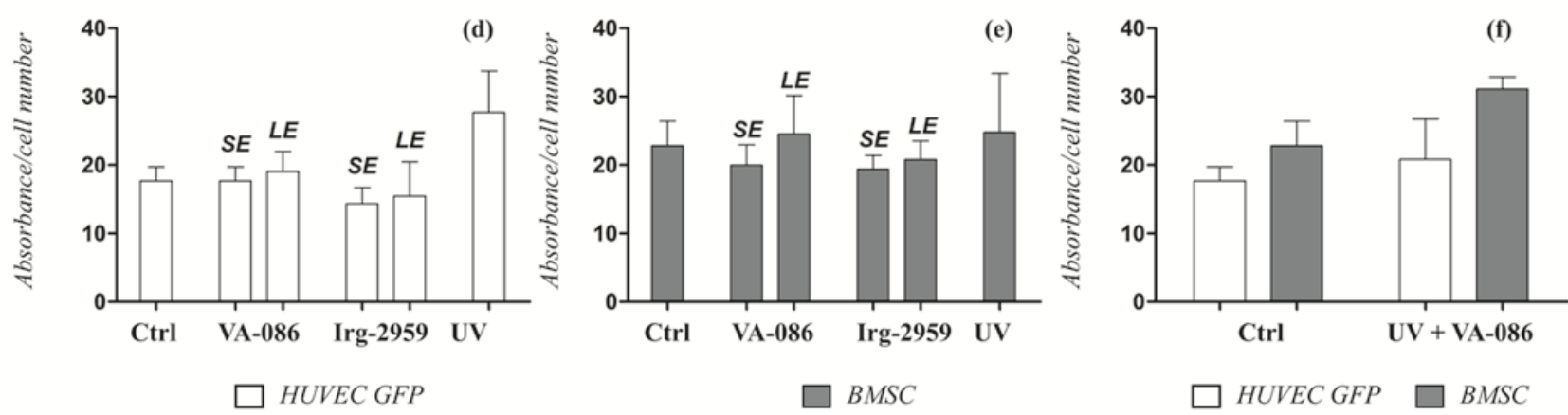

Figure 3. PI and UV cytotoxicity analyses. HUVEC GFP and BMSC 2D cultures were subjected to different photocrosslinking treatments to assess the PMP cytocompatibility. Single and combined effects of Short Exposure (SE, $4 \mathrm{hr}$ ) and Long Exposure (LE, 24hr) inactive PI molecules (VA-086 and Irg-2959) and UV ( $\lambda=385 \mathrm{~nm}$ for $3 \mathrm{~min}$ ) were tested 24 hours after the treatments. ((a), (b), (d) and (e)). For both cell types, the presence of both the PI molecules and the exposure to UV didn't cause any statistically significant reduction in cell number and specific metabolic activity compared to control. A reduction in HUVEC GFP number was detected for VA-086 LE though maintaining a high specific metabolic activity. ((c) and (f)) The combination of VA-086 and UV resulted favorable in terms of cell viability demonstrating the biocompatibility of PMP protocol. (Error bars: $\pm \mathrm{SD} ; \mathrm{n}=5$. All the reported comparisons are statistically significant for $\mathrm{p}<0.05)$.

\subsection{Cell encapsulation and distribution analysis}

HUVEC GFP cells were embedded in $10 \% \mathrm{w} / \mathrm{v}$ GelMA micropatterns to assess the potentiality of PMP protocol in achieving a 3D uniform cellular dispersion. The desired cellular microtopography, defined by the pattern geometry, was achieved resulting in a uniform distribution of HUVEC GFP dispersed along the straight GelMA micropatterns, as shown by the geometrical center positions of the cell nuclei (figure 4(a), (e) and (i)). For all the concentrations tested, cells preserved a tridimensional distribution along the whole channel after the photopolymerization, resulting homogenously dispersed within both the gel length (figure 4(b), (f) and (j)) 
and width (figure 4(c), (g) and (k)). The analysis of cells distribution along the microgels height (figure 4(d),

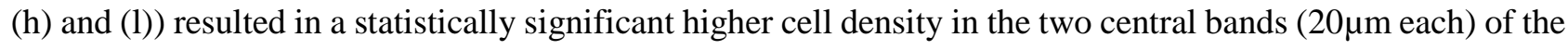
channels ( $\mathrm{p}<0.05$ ), probably due to the flow dynamics during the solution injection.

3D distribution

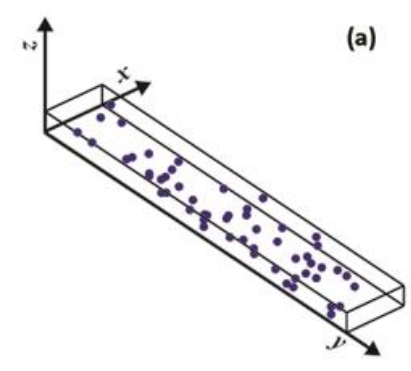

(a)

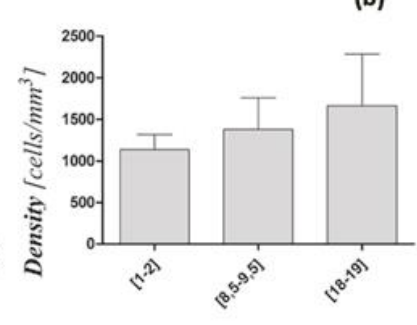

$\boldsymbol{y}[\mathrm{mm}]$
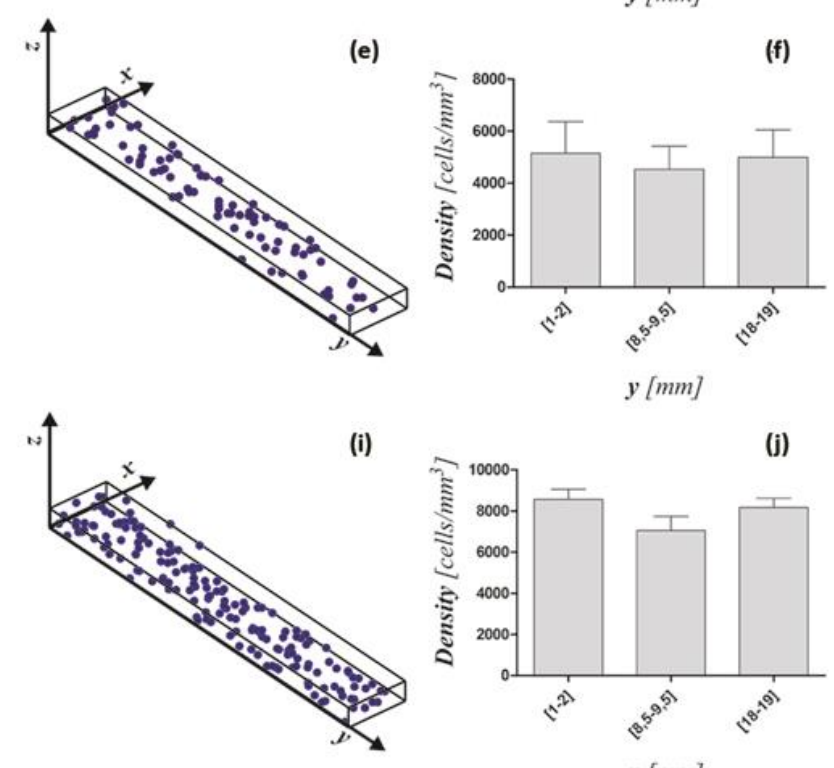

Width distribution

(c)

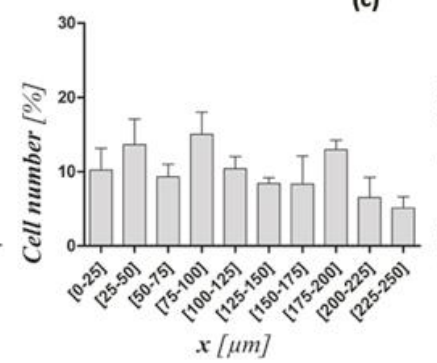

(g)

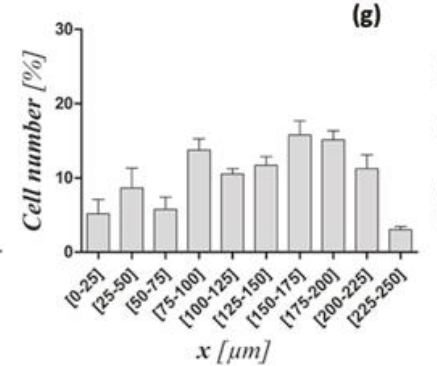

(k)

$y[\mathrm{~mm}]$

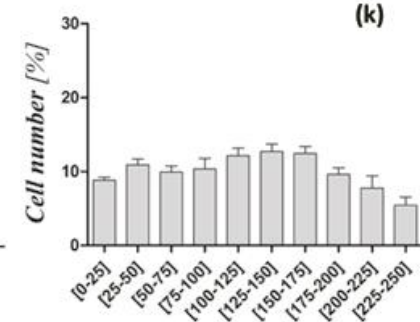

$x[\mu m]$
Height distribution

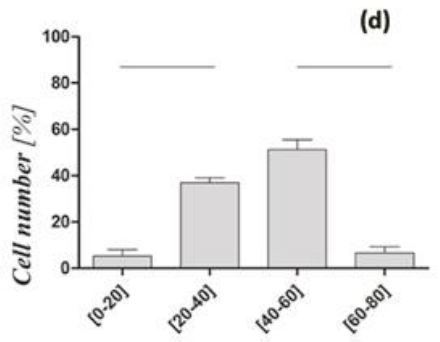

$z[\mu m]$

(h)

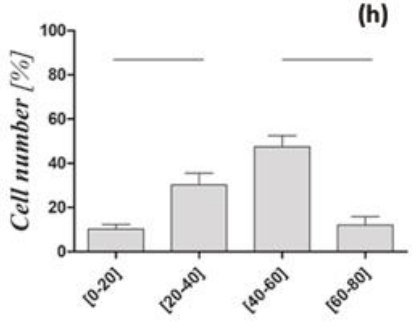

$z[\mu m]$

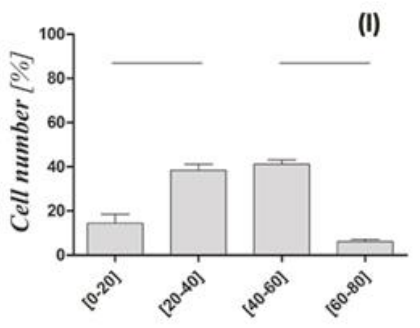

$z[\mu m]$

Figure 4. Cells spatial distribution. Positions of cell nuclei geometrical centers at densities of 1,5 and $10 \times 10^{6}$ cell ml $^{-1}$ were plotted from data obtained by analyzing confocal images of the DAPI stained cell-laden micropatterns at day 0 . Cells were uniformly distributed along the channels length ((b), (f) and (j)) and width ((c), (g) and (k)). While a higher cell density was detected in the two central bands $(\mathrm{l}=20 \mu \mathrm{m})$ of the channels height $((\mathrm{d})$, (h) and (l)), the overall cell distribution resulted 3D uniform within the micropatterns ((a), (e) and (i)). (Error bars: $\pm S D ; n=3$. All the reported comparisons are statistically significant for $\mathrm{p}<0.05$ ).

\subsection{Cell culture within hydrogel micropatterns}

For a preliminary biological validation of the PMP protocol, HUVEC GFP (figure 5(a) and (c)) and BMSCs (figure 5(b) and (d)) were encapsulated within GelMA micropatterns at a cell density of 10 and $3 \times 10^{6}$ cells $\mathrm{ml}^{-1}$ respectively, and their behavior was recorded over a 7 days culture period. After $24 \mathrm{~h}$ of culture both cell types started to actively elongate within the GelMA hydrogels. At day 3, both types of cells started to migrate from the center to the surface of the patterns. The maximum cell spreading was reached by day 7 of culture (figure 5(c) and (d)), when the cells completely covered the pattern surfaces. While HUVEC GFP cells created network-like structures, preferentially near the outer surface of the hydrogel, BMSCs showed a tendency to align along the main axis of the microstructures. 

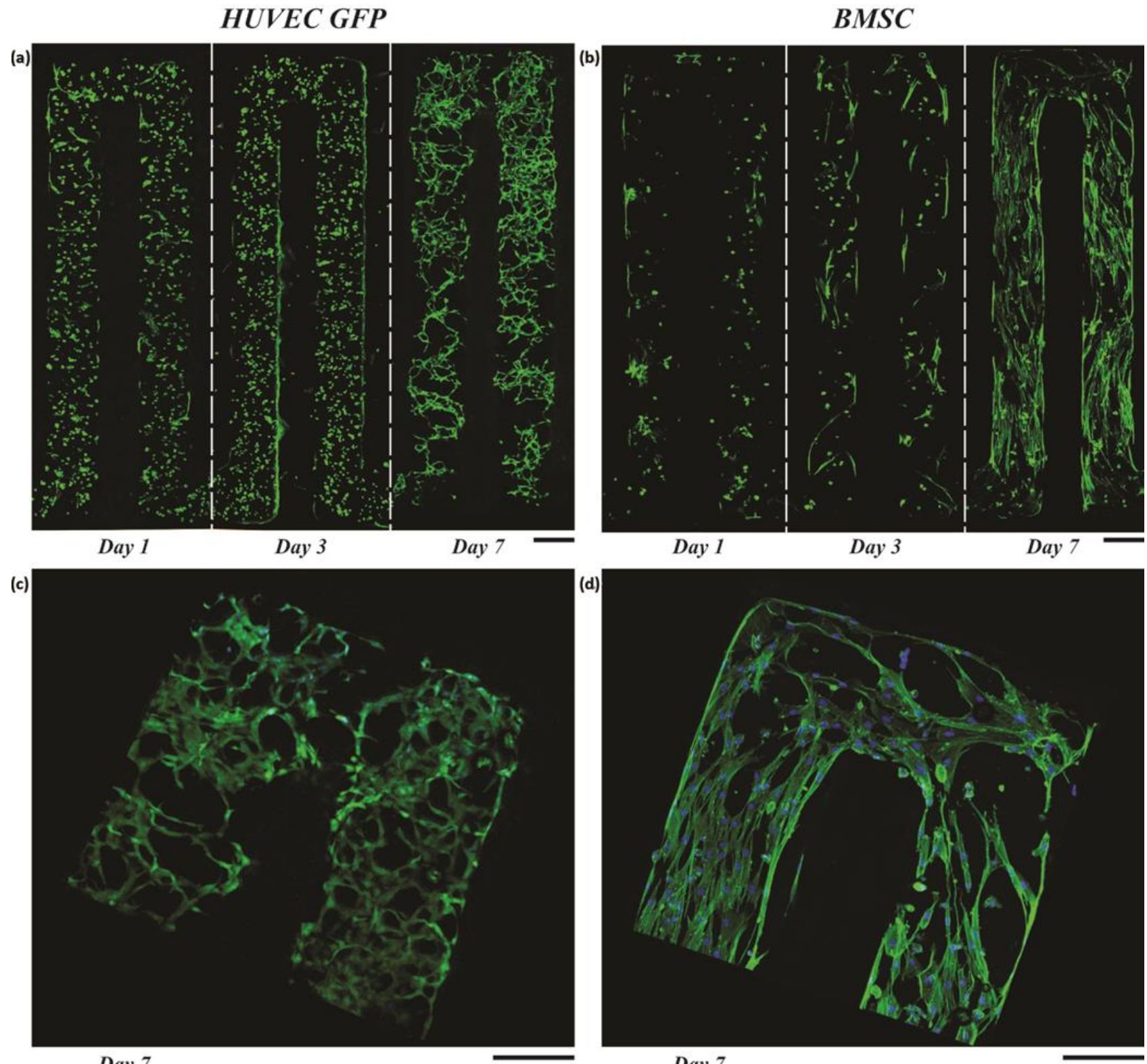

Day 7

Day 7

Figure 5. Cell behavior within hydrogel micropatterns. HUVEC GFP ((a) and (c)) and BMSC ((b) and (d)) were embedded within GelMA micropatterned hydrogels by PMP and cultured for 7 days. ((a) and (b)) The day 1 cell rounded morphology gave way at day 3 to a migration of both cell types from the center to the surface of the patterns, reaching the maximum cell spreading by day 7 of culture. (Representative images of F-actin stained samples; $\mathrm{z}$ step $=5.48 \mu \mathrm{m}$. Scale bar $=250 \mu \mathrm{m}$.). At day 7, HUVEC GFP created network-like structures preferentially near the outer surface of the hydrogel (c), while BMSCs showed a tendency to align along the main axis of the microstructures (d). (Representative F-actin/DAPI stained 3D projections; $\mathrm{z}=80.22 \mu \mathrm{m}$. Scale bar $=250 \mu \mathrm{m}$.).

\section{Discussion}

The design of engineered constructs and in vitro models structurally and functionally mimicking native tissues requires control over cellular distribution and organization within a 3D microenvironment. The design of engineered constructs and in vitro models structurally and functionally mimicking native tissues requires control over cellular distribution and organization within a 3D microenvironment. While some approaches, such as tissue morphogenesis studies, relies on the cells ability to re-organized themselves forming patterns on 
their own [40], there are other applications in which the capability to guide cell patterning in 3D is crucial to obtain functional tissue-like constructs [24]. In the last decade, advances in microtechnologies and definition of novel biomaterial compositions led to the development of microscale approaches for tailoring in vitro the microarchitecture around cells $[8,41]$. Three are the main requirements for the realization of 3D microstructured cell-populated constructs: $i$ ) the cytocompatibility of the fabrication/embedding protocol, $i i$ ) the ability to control the microenvironment in size and shape over the three dimensions, and iii) the possibility to distribute cells in a uniform and controlled fashion within the microenvironment. Several attempts for matching these requisites have been reported [5, 42-44]. Lately, the most promising approaches tried to combine the high spatial resolution achievable through photo and soft lithographic techniques with the unique affinity of hydrogels with several cell embedding approaches [12, 15, 16, 23].

Within this context, we presented an innovative Photo-mold Patterning (PMP) protocol aiming to meet biocompatibility and microarchitectural control requirements in a simple and cost effective way. The PMP technique enables for fast and controlled cell embedding within 3D micropatterns through the exploitation of standard PDMS soft lithography and an innovative low toxicity hydrogel photopolymerization approach. Indeed, unlike standard photopatterning methods, the PMP approach is based on a recently investigated lowtoxicity photoinitiator molecule [32] featuring an absorption peak in the near UV region (at around 380nm). As previously shown [31], cell damages from UV light exposure strictly depends on source intensity and emitting spectrum. While short UV wavelengths (UVB range) consistently damage cells even at low energy levels (in the order of $\mathrm{mJ} / \mathrm{cm}^{2}$ ), the shifting to higher wavelengths dramatically reduces the incidence of cell death. A low cost easily commercially available $385 \mathrm{~nm}$ LED was used in this study as light source, and specific experiments showed negligible effects in terms of cytotoxicity. Furthermore, the chosen photoinitiator molecule (VA-086), activated at this wavelength [32], was demonstrated to minimally affect cell viability both in its inactive and radical forms. These results confirmed that VA-086 is a valid alternative to other widely used photoinitiator molecules [45], by ensuring a high cell survival rate even subsequent to long exposures. The PMP method was tested both on a continuous cell line (HUVEC GFP) and primary human BMSCs demonstrating the possibility to embed viable cells within 3D microgels, creating biocompatible and favorable environments for cell proliferation and spreading.

The ability of PMP protocol to replicate defined microarchitectures through hydrogel photopolymerization was also demonstrated. Indeed, 3D structures obtained by PMP presented a geometrical resolution comparable to that offered by traditional mold patterning methods [16, 17]. In fact, the PMP protocol exploits UVA-transparent PDMS micromolds as physical constraints for cell-laden prepolymer. This leads to micrometrically resolved structures guaranteeing the desired 3D shape replication both in terms of layout and cross section independently from the light beam collimation [21]. This was demonstrated starting from two different prepolymer types (a natural and synthetic one) showing how the PMP protocol represents a versatile tool for controlling the 3D geometry of different methacrylated hydrogels in an effective and low cost way. Well-established multilayer photo and soft lithographic techniques expand the range of PMP feasible geometries, allowing to obtain multiple shapes and heights for channels within the same mold as detailed in 
the figure S1 and S2 of SI [46]. PMP can also be used as a flexible tool to combine different cells (and potentially materials) within features contained in the same mold (figure S3 of SI). This can thus be considered a simple tool to design and pattern complex 3D geometries with the aim of replicating in vivo-like microarchitectures within a single step procedure (see figure S1 and S3 of SI). Starting from the shown potentialities, further investigations are currently being pursued to implement a reliable multi-steps PMP methodology aiming to expand the feasibility to multi-layer geometries.

Moreover, the exploitation of a light-initiated polymerization approach enables for a fast cell immobilization within the matrices, generating micrometrically resolved hydrogel patterns upon a 3 minutes exposure to a UVA LED source. Such exposure period was compatible with the achievement of uniform 3D cell dispersions within the structures for the three encapsulation cell densities considered. Shorter crosslinking periods (within tens of seconds) could be achieved by exposing the prepolymer to higher light intensities. However, we noticed that at higher optical powers bubbles were generating within the structures thus compromising either the pattern spatial definition or integrity. Hence, PMP represents a valid tool to create homogenously populated cell-laden constructs able to spatially recapitulate the native 3D cell-cell and cellECM interactions.

As a remarkable advantage, the presented PMP protocol needs a minimal quantity of reagents and cells for micropatterns formation, being only required the amount of prepolymer to completely fill the mold channels. This advantage becomes more relevant in case two or more cell types and/or materials are considered as fillers of different features of the same mold (figure S3 of Supplementary Information). Additionally, due to the polystyrene transparency to UVA, the PMP can be fully performed under sterile conditions, housing the samples in culture petri dishes during the entire protocol. It is worth noticing that to the best of our knowledge this is the first time photopolymerizable cell-laden micropatterns are obtained through the use of a LED as light source.

\section{Conclusion}

In the present study we introduce a novel protocol to generate 3D hydrogel micropatterns with high geometrical resolution and user- defined size and shape. The method, named Photo-mold Patterning (PMP), combines hydrogels photopolymerization and PDMS stamps used as micromolds. The PMP approach allows uniform cell inclusion within micropatterns, creating cytocompatible and 3D geometrically controlled environments favorable for cells growth, migration and spreading. In this perspective, PMP could become a simple tool for designing accurate in vitro platforms aiming at recapitulating and better understanding physiologically relevant issues at a microscale level. Further investigations are needed to exploit the PMP potentialities for the replication of multi cellular 3D microenvironments with tailored mechanical (different composition hydrogels) and biochemical (different hydrogel functionalization) properties.

\section{Acknowledgments}


This study was partially supported by Fondazione Cariplo, Grant No. 2008- 2531, and by the Italian Ministry of Health. The authors would like to acknowledge Ing. Giuseppe Talò for his valuable help in the electrical setup configuration and Dr. Giulio Simonutti (Istituto Nazionale Genetica Molecolare - INGM, Milano, Italy) for the valuable support and expertise in confocal microscopy.

\section{References}

[1] Liu Tsang V and Bhatia S N 2004 Three-dimensional tissue fabrication Adv. Drug. Deliv. Rev. 11 1635-47

[2] Titmarsh D M, Chen H, Wolvetang E J and Cooper-White J J 2012 Arrayed cellular environments for stem cells and regenerative medicine Biotechnol. J. n/a-n/a

[3] Greiner A M, Richter B and Bastmeyer M 2012 Micro-Engineered 3D Scaffolds for Cell Culture Studies Macromol. Biosci. 10 1301-14

[4] Griffith L G and Swartz M A 2006 Capturing complex 3D tissue physiology in vitro Nat. Rev. Mol. Cell Biol. 3 211-24

[5] Fedorovich N E, Schuurman W, Wijnberg H M, Prins H-J, van Weeren P R, Malda J, Alblas J and Dhert W J A 2012 Biofabrication of Osteochondral Tissue Equivalents by Printing Topologically Defined, CellLaden Hydrogel Scaffolds Tissue Eng. C-Meth. $133-44$

[6] Kaji H, Camci-Unal G, Langer R and Khademhosseini A 2011 Engineering systems for the generation of patterned co-cultures for controlling cell-cell interactions BBA-Gen. Subjects $3239-50$ Khetan S and Burdick J A 2011 Patterning hydrogels in three dimensions towards controlling cellular interactions Soft Matter 3 830-8

[8] Gauvin R, Parenteau-Bareil R, Dokmeci M R, Merryman W D and Khademhosseini A 2012 Hydrogels and microtechnologies for engineering the cellular microenvironment Wiley Interdiscip. Rev. Nanomed. Nanobiotechnol. 3 235-46

[9] Geckil H 2010 Engineering hydrogels as extracellular matrix mimics Nanomedicine 469-84

[10] Tibbitt M W and Anseth K S 2009 Hydrogels as extracellular matrix mimics for 3D cell culture Biotechnol. Bioeng. 4 655-63

[11] Zhu J and Marchant R E 2011 Design properties of hydrogel tissue-engineering scaffolds Expert Rev. Med. Devic. 5 607-26

[12] Huang G Y, Zhou L H, Zhang Q C, Chen Y M, Sun W, Xu F and Lu T J 2011 Microfluidic hydrogels for tissue engineering Biofabrication 1012001

[13] Zhang L, Yuan T, Guo L and Zhang X 2012 An in vitro study of collagen hydrogel to induce the chondrogenic differentiation of mesenchymal stem cells J. Biomed. Mater. Res. A 10 2717-25

[14] Nichol J W, Koshy S T, Bae H, Hwang C M, Yamanlar S and Khademhosseini A 2010 Cell-laden microengineered gelatin methacrylate hydrogels Biomaterials 21 5536-44

[15] Yeh J, Ling Y, Karp J M, Gantz J, Chandawarkar A, Eng G, Blumling Iii J, Langer R and Khademhosseini A 2006 Micromolding of shape-controlled, harvestable cell-laden hydrogels Biomaterials 31 5391-8

[16] Trkov S, Eng G, Di Liddo R, Parnigotto P P and Vunjak-Novakovic G 2010 Micropatterned threedimensional hydrogel system to study human endothelial-mesenchymal stem cell interactions J. Tissue Eng. Regen. M. 3 205-15

[17] Raghavan S, Nelson C M, Baranski J D, Lim E and Chen C S 2010 Geometrically Controlled Endothelial Tubulogenesis in Micropatterned Gels Tissue Eng. A 7 2255-63

[18] Ling Y, Rubin J, Deng Y, Huang C, Demirci U, Karp J M and Khademhosseini A 2007 A cell-laden microfluidic hydrogel Lab Chip 6756

[19] Ehrbar M, Sala A, Lienemann P, Ranga A, Mosiewicz K, Bittermann A, Rizzi S C, Weber F E and Lutolf M P 2011 Elucidating the Role of Matrix Stiffness in 3D Cell Migration and Remodeling Biophys. J. 2 28493

[20] Ifkovits J L and Burdick J A 2007 Review: Photopolymerizable and Degradable Biomaterials for Tissue Engineering Applications Tissue Eng. 10 2369-85

[21] Liu V and Bhatia S 2002 Three-Dimensional Photopatterning of Hydrogels Containing Living Cells Biomed. Microdevices 4 257-66

[22] Du Y, Ghodousi M, Qi H, Haas N, Xiao W and Khademhosseini A 2011 Sequential Assembly of CellLaden Hydrogel Constructs to Engineer Vascular-Like Microchannels Biotechnol. Bioeng. 7 1693-703

[23] Aubin H, Nichol J W, Hutson C B, Bae H, Sieminski A L, Cropek D M, Akhyari P and Khademhosseini A 2010 Directed 3D cell alignment and elongation in microengineered hydrogels Biomaterials 27 6941-51 Tsang V L, Chen A A, Cho L M, Jadin K D, Sah R L, DeLong S, West J L and Bhatia S N 2007 Fabrication of 3D hepatic tissues by additive photopatterning of cellular hydrogels FASEB J. 3 790-801 
Lu T, Li Y and Chen T 2013 Techniques for fabrication and construction of three-dimensional scaffolds for tissue engineering International journal of nanomedicine 337-50

[26] Liu A, Bhatia, S.N. 2002 Three- Dimesional Photopatterning of Hydrogels Containing Living Cells Biomed. Microdevices 4 257-66

Bryant S J, Nuttelman C R and Anseth K S 2000 Cytocompatibility of UV and visible light photoinitiating systems on cultured NIH/3T3 fibroblasts in vitro J. Biomat. Sci.-Polym. E. 5 439-57

Mironi-Harpaz I, Wang D Y, Venkatraman S and Seliktar D 2012 Photopolymerization of cellencapsulating hydrogels: Crosslinking efficiency versus cytotoxicity Acta Biomater. 5 1838-48

Williams C G, Malik A N, Kim T K, Manson P N and Elisseeff J H 2005 Variable cytocompatibility of six cell lines with photoinitiators used for polymerizing hydrogels and cell encapsulation Biomaterials 11 12118

Fairbanks B D, Schwartz M P, Bowman C N and Anseth K S 2009 Photoinitiated polymerization of PEGdiacrylate with lithium phenyl-2,4,6-trimethylbenzoylphosphinate: polymerization rate and cytocompatibility Biomaterials 35 6702-7

Tuchinda C, Lim H W, Strickland F M, Guzmán E A and Wong H K 2007 Comparison of broadband UVB, narrowband UVB, broadband UVA and UVA1 on activation of apoptotic pathways in human peripheral blood mononuclear cells Photodermatol. Photoimmunol. Photomed. $12-9$

Rouillard A D, Berglund C M, Lee J Y, Polacheck W J, Tsui Y, Bonassar L J and Kirby B J 2011 Methods for Photocrosslinking Alginate Hydrogel Scaffolds with High Cell Viability Tissue Eng. C-Meth. 2 173-9 Chandler E M, Berglund C M, Lee J S, Polacheck W J, Gleghorn J P, Kirby B J and Fischbach C 2011 Stiffness of photocrosslinked RGD-alginate gels regulates adipose progenitor cell behavior Biotechnol. Bioeng. 7 1683-92

Xia Y and Whitesides G M 1998 Soft Lithography Angewandte Chemie International Edition 5 550-75

Moeller H C, Mian M K, Shrivastava S, Chung B G and Khademhosseini A 2008 A microwell array system for stem cell culture Biomaterials 6 752-63

Hancock M J, Piraino F, Camci-Unal G, Rasponi M and Khademhosseini A 2011 Anisotropic material synthesis by capillary flow in a fluid stripe Biomaterials 27 6493-504

Piraino F, Camci-Unal G, Hancock M J, Rasponi M and Khademhosseini A 2012 Multi-gradient hydrogels produced layer by layer with capillary flow and crosslinking in open microchannels Lab Chip 3 659-61

Fedorovich N E, Swennen I, Girones J, Moroni L, van Blitterswijk C A, Schacht E, Alblas J and Dhert W J A 2009 Evaluation of Photocrosslinked Lutrol Hydrogel for Tissue Printing Applications Biomacromolecules 7 1689-96

Hutson C B, Nichol J W, Aubin H, Bae H, Yamanlar S, Al-Haque S, Koshy S T and Khademhosseini A 2011 Synthesis and Characterization of Tunable Poly(Ethylene Glycol): Gelatin Methacrylate Composite Hydrogels Tissue Engineering Part A 13-14 1713-23

Karoly J, Cyrille N, Francoise M, Keith M, Gordana V-N and Gabor F 2010 Tissue engineering by selfassembly and bio-printing of living cells Biofabrication 2022001

Gauvin R and Khademhosseini A 2011 Microscale Technologies and Modular Approaches for Tissue

Engineering: Moving toward the Fabrication of Complex Functional Structures Acs Nano 6 4258-64
Nikkhah M, Edalat F, Manoucheri S and Khademhosseini A 2012 Engineering microscale topographies to control the cell-substrate interface Biomaterials 21 5230-46

Jin L, Wang T, Zhu M-L, Leach M K, Naim Y I, Corey J M, Feng Z-Q and Jiang Q 2012 Electrospun Fibers and Tissue Engineering J. Biomed. Nanotechnol. 1 1-9

Park S A, Lee S H and Kim W 2011 Fabrication of hydrogel scaffolds using rapid prototyping for soft tissue engineering Macromol. Res. 7 694-8

Fedorovich N E, Oudshoorn M H, van Geemen D, Hennink W E, Alblas J and Dhert W J A 2009 The effect of photopolymerization on stem cells embedded in hydrogels Biomaterials 3 344-53

[16] Rasponi M, Piraino F, Sadr N, Laganà M, Redaelli A and Moretti M 2011 Reliable magnetic reversible assembly of complex microfluidic devices: fabrication, characterization, and biological validation Microfluid Nanofluid 5 1097-107 